\title{
EFECTO DEL FUEGO SOBRE EL BOSQUE, LA DISPONIBILIDAD DE NUTRIENTES Y LA MATERIA ORGÁNICA EN EL SUELO.
}

\author{
RÓMULO SANTELICES (*) y CREIGHTON LITTON (**)
}

(*) Ingeniero Forestal.

(**) M. Sc. Ingeniero Forestal. Universidad Católica del Maule, Casilla 617, Talca, Chile.

\section{RESUMEN}

El uso del fuego es una costumbre arraigada desde hace mucho tiempo en Chile. Básicamente, se le ha utilizado para la habilitación de terrenos con fines agropecuarios o para la eliminación de residuos de cosechas agrícolas o forestales. A pesar de su generalizado empleo como herramienta del manejo, es común que se desconozcan todos los efectos que produce su uso sobre el sitio. En base a una revisión bibliográfica se intenta describir el efecto que tiene el fuego sobre el ecosistema boscoso, la disponibilidad de nutrientes y la materia orgánica en el suelo.

Palabras Clave: Fuego, Materia orgánica, Disponibilidad de nutrientes, Carbono, Nitrógeno.

\section{ABSTRACT}

The use of fire in Chile has been an inherent custom for many years. Basically, fire has been utilized for clearing agricultural and grazing lands and for the elimination of the harvesting residues from forestry and agricultural activities. The widely accepted application of fire as a management tool suggests a lack of knowledge about the effects that it produces on the site. The effect of fire on the forest ecosystem, the availability of nutrients, and the organic matter in the soil is described in reference to existing studies.

Keywords: Fire, Organic matter, Nutrient availability, Carbon, Nitrogen. 


\section{INTRODUCCIÓN}

La eliminación de residuos originados de las cosechas de productos agrícolas y forestales, se ha realizado en Chile tradicionalmente utilizando el fuego. Esta práctica ha sido muy difundida, en forma de quemas controladas, porque es relativamente sencilla de realizar y no se requiere de muchos recursos para su implementación. Además, en forma posterior se facilitan las faenas de establecimiento del nuevo cultivo.

El fuego también ha sido utilizado en la habilitación de terrenos para usos agrícolas y ganaderos, e incluso para facilitar la cosecha de algunas especies de bosques naturales. En muchos casos, estas prácticas se han realizado sin las debidas precauciones y así se han generado incendios de gran intensidad y que han llegado a durar incluso algunos meses.

Durante mucho tiempo se ha pensado, principalmente desde el punto de vista económico, que la utilización del fuego trae consigo más ventajas que desventajas. No obstante, en la actualidad existe la clara convicción de que la alteración de los ecosistemas está íntimamente relacionada con la pérdida de la productividad de ellos, fundamentalmente por el daño que se causa al suelo, ya sea en el mediano o largo plazo. Por ello, cada vez son más estudiados los procesos de transformación de los recursos naturales, incluyendo el efecto del fuego en la eliminación de los residuos de las cosechas.

El manejo sostenible de los recursos naturales adquiere cada vez mayor fuerza y en este sentido resulta fundamental el mantenimiento de la productividad de los ecosistemas. Por ello, es importante estudiar el efecto que tienen las diversas actividades del ciclo productivo en tal aspecto, en donde el uso del fuego muchas veces juega un rol gravitante.

A través del presente trabajo se analiza el efecto que tiene el fuego sobre el ecosistema boscoso, con énfasis en la disponibilidad de nutrientes y el efecto sobre la materia orgánica del suelo.

\section{EFECTOS DEL FUEGO SOBRE EL BOSQUE}

El efecto del fuego sobre el bosque puede ser perjudicial o beneficioso, dependiendo de dónde, cuándo y cómo se aplique, aunque generalmente es, en Chile, un factor destructivo de la vegetación (DONOSO, 1992). SPURR y BARNES (1980) y PRITCHETT y FISHER (1987) señalan que su influencia sobre el ecosistema boscoso se manifiesta en los siguientes aspectos:

- Propiedades físicas y químicas del sitio.

- Acumulación de materia seca. 
- Adaptaciones genéticas de las especies.

- Establecimiento, desarrollo, composición y diversidad de las especies. De este modo determina, a menudo, las relaciones en la comunidad, especialmente en las sucesiones.

- Hábitat y poblaciones de vida silvestre.

- Presencia y abundancia de insectos forestales, parásitos y hongos.

- Presencia y abundancia de la microflora y microfauna en el suelo.

Los resultados del fuego sobre el bosque pueden ser considerados como efectos indirectos o directos.

Los efectos indirectos se relacionan principalmente con los cambios de vegetación. Incendios muy intensos matarán la mayoría o toda la vida vegetal sobre la superficie del suelo. El cambio sucesional de la vegetación tenderá a ser con especies heliófitas, de semilla liviana, sin latencia, cuya germinación es inducida por el calor, y capaces de colonizar el área quemada; también puede darse con las especies que han desarrollado la capacidad de reproducirse vegetativamente a partir de rebrotes de los tocones o raíces. Muchas leguminosas caen en esta categoría, y la abundancia de estas y otras plantas fijadoras de nitrógeno es a menudo incrementada por la quema (SPURR y BARNES, 1980; DONOSO, 1992).

Otro efecto indirecto del fuego, es la influencia que ejerce sobre la flora y la fauna del suelo, que actúan descomponiendo la materia orgánica, fijando nitrógeno y proporcionándole aireación. La secuela de la quema en estos organismos va a depender de la intensidad del fuego, la profundidad a la cual llega y el tiempo que dure, el tipo de suelo, y la naturaleza de la vegetación o combustible existente (SPURR y BARNES, 1980).

Producto de la quema también se disminuye la humedad y la capacidad de infiltración del suelo, aumentando el escurrimiento superficial y la erosión (ALTIERI y RODRIGUEZ, 1975). Al incrementar el escurrimiento superficial y la erosión, además se produce un lavado del lecho de cenizas, transportando los elementos minerales que en ella se encuentran; producto de la erosión también se produce una pérdida, junto con el suelo, de elementos minerales (WELLS et al., 1979; LEITCH et al., 1983; PEÑA, 1994).

Los efectos directos se consideran al daño, y generalmente la muerte, de los organismos que conforman el ecosistema forestal. Además de la destrucción de los árboles, el fuego también causa interferencias en los procesos fisiológicos, siendo el más grave el efecto sobre la fotosíntesis, causado por los llamados incendios de copa. El fuego al destruir parte de la copa de los árboles, disminuye la superficie foliar y en consecuencia también merma la capacidad de fotosíntesis del árbol, con la consiguiente pérdida de crecimiento. De acuerdo a lo señalado por HAWLEY y SMITH (1972), numerosos estudios indican que al reducirse la copa viva entre un 25 y $30 \%$, el 
crecimiento disminuye considerablemente. Incendios de copa muy severos, fácilmente pueden llegar a destruir más de un tercio de la copa del árbol.

Otros daños directos son las lesiones causadas en la corteza y la zona cambial, que no merman el crecimiento, pero si disminuyen el valor comercial de la madera. La intensidad del daño será mayor en árboles jóvenes y en aquellas especies que no hayan desarrollado mecanismos de defensa contra los incendios, tales como cortezas gruesas (SPURR y BARNES, 1980).

También se consideran efectos directos la quema de la materia orgánica sobre el suelo mineral y el calentamiento de las capas superficiales del suelo. Como consecuencia de la quema de la materia orgánica, se libera a la atmósfera dióxido de carbono, nitrógeno y cenizas, y se depositan minerales en la forma de cenizas, alterando el ciclo de nutrientes. Si la intensidad del fuego es alta, el nitrógeno se volatiliza y se pierde. Además se promueve la solubilización de los compuestos $\mathrm{Ca}, \mathrm{P}$ y $\mathrm{K}$, lo cual incrementa la cantidad de minerales disponible, pero sólo en forma temporal, ya que rápidamente son lixiviados por las lluvias posteriores. Además por efecto de la liberación de las bases se aumenta el pH del suelo (SPURR y BARNES, 1980; DONOSO, 1992).

\section{Causas y Tipos de Fuegos}

El fuego puede originarse por causas naturales o antrópicas. Estos últimos pueden manifestarse como quemas prescritas o incendios fuera de control. En otras latitudes, son frecuentes los incendios naturales como modeladores de la estructura del paisaje y la composición de los ecosistemas. Sin embargo, en Chile no tienen casi ninguna relevancia. La gran mayoría de los incendios que se producen en nuestro país son causados por la acción directa del hombre, muchas veces debido a una quema prescrita mal efectuada.

De acuerdo al nivel al cual se produce la quema, se pueden encontrar diferentes tipos de incendios, teniendo cada uno de ellos diferentes efectos en las propiedades del suelo y del bosque.

El tipo de incendio más común es el fuego superficial. Se desarrolla a nivel del suelo, consumiendo la litera y el humus, matando plantas herbáceas y arbustos, y chamuscando la base y la copa de los árboles. La intensidad y el daño que causen va a depender del combustible acumulado en el suelo. El mayor estrago lo causan en árboles jóvenes de cualquier especie.

Producto de un fuego superficial y por la acción del viento se pueden chamuscar y posteriormente encender las copas de los árboles, generando los llamados fuegos de copa. Estos se desarrollan de una copa a otra, sobre todo en rodales densos, matando muchos árboles en este trayecto. Las coníferas son más susceptibles a los incendios de copa por tener un follaje más combustible que las latifoliadas. Sin embargo, es poco 
frecuente que se produzcan en forma muy extensa y devastadora. Dependiendo de la porción de copa que se queme, va a ser el daño que produzcan en el árbol.

En suelos profundos y con abundante materia orgánica, en forma posterior a una sequía se pueden generar los llamados fuegos subterráneos, que queman las acumulaciones de materia orgánica sobre el suelo mineral. Se caracterizan por quemar bajo la superficie, ser muy inflamables, y porque pueden matar a todas las plantas que desarrollan su sistema radicular en la materia orgánica. Tienen una combustión muy lenta y usualmente generan altas temperaturas. Tienden a ser muy persistentes y sirven como reignición a los fuegos superficiales. Sobre este tipo de fuego BARBOUR et al. (1987) opinan que destruyen el $100 \%$ del suelo y llevan al ecosistema a una fase de sucesión primaria, necesitándose 10.000 años para su recuperación.

\section{Efecto del Fuego sobre la Disponibilidad de Nitrógeno, Fósforo y Potasio en el Suelo}

Como ya se ha mencionado, el fuego puede influir en la cantidad de nitrógeno que se encuentra en el suelo de varias formas. Una de ellas es alterando aquel que se encuentra en la materia orgánica sobre el suelo, reduciéndola a un lecho de cenizas e induciendo a una rápida mineralización inicial del nitrógeno (FRANCKE, 1989 y 1992). Sin embargo, el nitrógeno en forma de nitrato es más soluble y fácilmente puede ser lixiviado a capas más profundas del suelo (OPAZO, 1994). Además, por efecto del escurrimiento superficial y del viento el nitrógeno contenido en el lecho de cenizas puede ser movilizado hacia otros lugares, produciéndose las consecuentes pérdidas.

El lecho de cenizas es rico en bases intercambiables y eleva el $\mathrm{pH}$ del suelo, con esto se favorece la acción de la flora bacteriana del suelo y el proceso de nitrificación (WELLS ㄴ. al., 1979; SPURR y BARNES, 1980; DONOSO, 1992).

Por efecto del calentamiento del suelo el nitrógeno se volatiliza, perdiéndose hacia la atmósfera. El grado de volatilización va a depender de la intensidad del fuego. KNIGHT (1966) cit. p. SPURR y BARNES (1980), trabajando en bosques de Pseudotsuga menziesii (Mirb.) Franco en Estados Unidos, encontró que no existían pérdidas de nitrógeno en los suelos calentados a $200^{\circ} \mathrm{C}$, un $25 \%$ de pérdida con $300^{\circ} \mathrm{C}$, y un $64 \%$ de pérdida a los $700^{\circ} \mathrm{C}$. Estudios de laboratorio, realizados por WHITE et al. (1973) cit. p. WELLS et al. (1979), con muestras de bosques de Pinus ponderosa Dougl. ex Laws., confirman lo anterior, ya que las pérdidas de nitrógeno se produjeron sobre los $200^{\circ} \mathrm{C}$. Con temperaturas superiores a los $100^{\circ} \mathrm{C}$, se produce la destrucción total del mantillo y de gran parte de la materia orgánica, pero no se volatiliza el nitrógeno (DONOSO, 1992).

En quemas prescritas para el control de la competencia en bosques de Pinus spp. en Estados Unidos, se han estimado pérdidas, por efecto de la volatilización, que oscilan 
entre 112 y $140 \mathrm{~kg}$ de N/ha; y entre 10 y $20 \%$ (en caso extremo) del nitrógeno total en el ecosistema (WELLS, 1971; WELLS et al., 1979).

En quemas de residuos de la cosecha, que son de mayor intensidad y magnitud que las quemas prescritas para controlar la competencia, GRIER (1975) cit. p. WELLS et al. (1979) y SPURR y BARNES (1980) señala que en bosques de Pinus ponderosa Dougl. ex Laws. en el estado de Washington las pérdidas se han estimado en $907 \mathrm{~kg}$ de $\mathrm{N} / \mathrm{ha}$, lo que equivale a aproximadamente un $97 \%$ del nitrógeno originalmente en el piso del bosque y a la pérdida de dos tercios del contenido en el horizonte $A_{1}$ del suelo mineral.

Otros antecedentes sobre grandes pérdidas de nitrógeno por volatilización producto de la quema de residuos, indican valores de $750 \mathrm{~kg}$ de $\mathrm{N} / \mathrm{ha}$ en bosques de Pseudotsuga menziesii (Mirb.) Franco (YOUNGBERG y WOLLUM, 1976 cit. p. WELLS et al., 1979).

Con el objeto de analizar el efecto de la quema sobre la disponibilidad de nitrógeno y fósforo, y de las tasas de mineralización y nitrificación del nitrógeno, KUTIEL y SHAVIV (1989) estudiaron la combustión de suelos en laboratorio. Estos autores llegaron a la conclusión de que con fuegos con temperaturas de $600^{\circ} \mathrm{C}$ se produce una completa volatilización del $\mathrm{NH}_{4}$ y un incremento significativo en el pH, de 7,6 a 11,7. Bajo esas condiciones los procesos de amonificación y nitrificación fueron inhibidos. Inmediatamente después que el fuego alcanzó los $600^{\circ} \mathrm{C}$, se produjo una menor disponibilidad de fósforo, al compararlo cuando el fuego tenía $250^{\circ} \mathrm{C}$. Al quemar el suelo con plantas, se produjo una menor tasa de amonificación y nitrificación, pero aumentó la disponibilidad de fósforo en el suelo. El aumento del $\mathrm{pH}$ y de la cantidad de fósforo, que puede ser hasta 300\%, es temporal (KUTIEL y NAVEH, 1987).

El fuego produce en el corto plazo un aumento en la disponibilidad de todos los nutrientes (KUTIEL y NAVEH, 1987; MARION et al., 1991). En forma posterior al fuego, en el área quemada se desarrollan especies herbáceas que aprovechan esos nutrientes y posteriormente son devueltos al suelo poco a poco amortiguando la pérdida de ellos. Junto con ello, también el pH tiende a normalizarse (KUTIEL y NAVEH, 1987).

Para mantener la productividad de las áreas frecuentemente quemadas es necesario la adición de nitrógeno mediante procesos de fijación. La depositación atmosférica depende de la región y puede variar de 1 a $22 \mathrm{~kg} / \mathrm{ha}$ (LANDSBERG, 1986), lo que es insuficiente para balancear las pérdidas producto de la quema u otros mecanismos. Esto lleva a un cambio en la sucesión, cambiando la composición de las especies por aquellas capaces de fijar simbióticamente nitrógeno, como lo son muchas especies de leguminosas (WELLS et al., 1979).

En Chile se han realizado algunas experiencias para evaluar el efecto del fuego, ya sea por incendios o por la quema de residuos de la cosecha, sobre las propiedades del suelo. ALTIERI y RODRIGUEZ (1975), en uno de los primeros estudios efectuados, 
encontraron que la cantidad de nitrógeno soluble fue de $0,42 \%$ en un sector no quemado, contra $0,16 \%$ en un sector quemado, es decir, se redujo por efecto del fuego en más de un $60 \%$.

Al analizar y comparar dos áreas en el sur de Australia, una sometida a quemas frecuentes y otra sin quemar por un largo período, ADAMS et al. (1994) indicaron que los efectos del fuego se limitaron a los primeros $2 \mathrm{~cm}$ del suelo y que fue menor la actividad fosfática, la concentración de carbono, y la cantidad de nitrógeno, incluyendo aquel potencialmente mineralizable, en los suelos sometidos a quemas frecuentes. Por otra parte, en este mismo tipo de suelos, aumentó la concentración de fósforo inorgánico extraíble. Los suelos del área estudiada son de origen marino con comunidades arbustivas de la familia Ericaceae.

Para mantener la productividad del sitio es recomendable, después de la cosecha, distribuir los residuos en todo el terreno, para que de este modo se conserve la humedad, temperatura y nutrientes del suelo, y no eliminarlos con fuego; al utilizar la primera la técnica mencionada, y sobre todo en suelos arenosos, se produce una menor pérdida de nitrógeno (TORO, 1987).

Para evaluar el efecto del fuego en la disponibilidad de nitrógeno total, TORO en 1987 decidió comparar dos métodos de tratamientos de los residuos de la cosecha del bosque, en diferentes predios de la zona centro sur de Chile. Las técnicas utilizadas fueron las siguientes:

- quema de los residuos sin apilarlos, usando el método de encendido fuego en retroceso

- retiro de los residuos mayores a $3 \mathrm{~cm}$ de diámetro, sin efectuar quema

Después de seis meses de efectuados los tratamientos, concluyó que se produce una pérdida de alrededor de un $63 \%$ en un suelo arenoso, al interior de la VIII Región (Predio Maquehua); mientras que en otro arcilloso en la costa de la VIII Región (Predio La Colcha), fue de un $44 \%$. En todos los predios estudiados se produjo pérdida de nitrógeno, pero en un suelo franco arcillo arenoso de la VII Región (Predio San Pedro), ésta fue sólo de casi un $3 \%$. Esta menor pérdida podría explicarse porque en el predio de la VII Región la pluviometría es casi la mitad que en los predios de la VIII Región, razón por la cual podría producirse una menor lixiviación.

FRANCKE (1989, 1991 y 1992) al evaluar después de seis meses los ensayos antes analizados por TORO, es decir, un año después de instalados los experimentos, señala que con todos los tratamientos probados se produce una aumento en el contenido nitrógeno en el suelo, al compararlos con los valores de seis meses antes. Sin embargo, hace mención a la inconveniencia de realizar quemas en suelos arenosos y en suelos arcillosos analizar la posibilidad de realizar otro tipo de manejo de los residuos, como por ejemplo astillado del material. 
En un experimento en la Columbia Británica, al analizar el efecto en el suelo, después de dos años, de la cosecha a tala rasa de un bosque, en el cual los residuos en un sector fueron quemados y en otro no, FELLER y KIMMINS (1984) encontraron diferencias aun más apreciables que las de TORO y FRANCKE, señaladas anteriormente. En efecto, estos autores señalan que producto sólo de la cosecha a tala rasa, hubo una pérdida de nitrógeno equivalente a $245 \mathrm{~kg} / \mathrm{ha}$, mientras que al combinarse con la quema, la pérdida aumento a $1.293 \mathrm{~kg} / \mathrm{ha}$.

Muchos autores señalan que como consecuencia del fuego, ya sea en forma de quema o de incendio forestal, se promueve una liberación de las bases, razón por la cual se produce un aumento de calcio, potasio y fósforo (WELLS, 1971; TORO, 1987; SPURR y BARNES (1980; FRANCKE, 1989, 1991 y 1992; DONOSO, 1992). En el lecho de cenizas estos compuestos están disponibles para ser utilizados por las plantas, pero por el hecho de ser más solubles pueden ser, por efecto de las lluvias posteriores, rápidamente lixiviados o arrastrados por la escorrentía superficial.

\section{Efecto del Fuego sobre la Materia Orgánica y la Relación Carbono/Nitrógeno en el Suelo}

El efecto más obvio de la quema es la reducción del material que se encuentra en el piso del bosque. Por ejemplo, después de una quema realizada en invierno, se produjeron pérdidas de cerca de $3.000 \mathrm{~kg}$, de un total de $12.000 \mathrm{~kg}$. Sin embargo, la cantidad de materia orgánica total del suelo en el largo plazo no cambia significativamente porque aumenta la de los primeros $5 \mathrm{~cm}$ (WELLS, 1971). La cantidad de materia orgánica destruida en un incendio depende de la intensidad del fuego y a su vez esta lo hace de la cantidad de materia orgánica y de las condiciones climáticas. En un área quemada anualmente se producen pérdidas de entre 2.000 y $4.000 \mathrm{~kg}$ por hectárea y en un área quemada después de varios años sin la presencia del fuego es de 4.000 a $9.000 \mathrm{~kg} / \mathrm{ha}$ (PRITCHETT and FISHER, 1987).

Si las temperaturas alcanzadas en el suelo son inferiores a $200^{\circ} \mathrm{C}$, se produce un aumento en la materia orgánica, al menos en los primeros horizontes del suelo (TORO, 1987). Al incrementar la materia orgánica, proporcionalmente también lo hace el carbono. La cantidad de litera consumida varía con la intensidad del fuego, por ejemplo fuegos de alta intensidad $\left(300^{\circ} \mathrm{C}\right.$ sobre la superficie del suelo), consumen toda la litera; fuegos de mediana intensidad $\left(180\right.$ a $\left.300^{\circ} \mathrm{C}\right)$ consumen cerca de la mitad de la litera; y fuegos de baja intensidad, sólo chamuscan la litera (STARK, 1977 cit. p. KOZLOWSKI et al., 1991). En opinión de BARBOUR et al., (1987), con intensidades entre 200 y $300^{\circ} \mathrm{C}$ se destruye un $85 \%$ de la materia orgánica, lo que incide en una disminución de la capacidad de intercambio catiónico.

La quema de los residuos de una cosecha forestal de Pinus radiata D. Don, transforma casi en su totalidad el mantillo a un lecho de cenizas. Si esto sucede en suelos arcillosos, aumenta el contenido de materia orgánica en los primeros $20 \mathrm{~cm}$ de 
profundidad. En cambio, si los suelos son arenosos se producen severas pérdidas. Estas son mayores si se trabaja en suelos con pendientes superiores al $30 \%$ y precipitaciones de alrededor de $1.500 \mathrm{~mm}$ anuales (FRANCKE, 1991 y 1992).

FRANCKE (1989) entrega antecedentes sobre la relación carbono orgánico total (CT)/nitrógeno total (NT), para distintos tratamientos de los residuos de una cosecha de Pinus radiata D. Don, en diferentes condiciones de suelo, después de un año (Cuadros 1 y 2).

\section{Cuadro 1}

\section{RELACIÓN CT/NT EN PREDIO MAQUEHUA (SUELO ARENOSO)}

\begin{tabular}{|c|c|c|c|c|c|c|}
\hline \multirow{2}{*}{$\begin{array}{c}\text { Estrata } \\
(\mathrm{cm})\end{array}$} & \multicolumn{7}{|c|}{ Tratamiento } \\
\cline { 2 - 7 } & $\mathrm{T} 1$ & $\mathrm{~T} 2$ & $\mathrm{~T} 3$ & $\mathrm{~T} 4$ & $\mathrm{~T} 5$ & T6 \\
\hline Orgánica & 15,6 & 23,9 & 24,7 & 32,9 & 19,7 & 21,8 \\
\hline $0-5$ & 12,0 & 22,0 & 17,1 & 16,7 & 14,3 & 13,3 \\
\hline $5-10$ & 12,5 & 17,5 & 11,7 & 11,7 & 14,3 & 12,5 \\
\hline $10-20$ & 11,4 & 10,0 & 15,0 & 15,0 & 8,3 & 11,7 \\
\hline
\end{tabular}

T1: Residuos apilados - Fuego en retroceso

T2: Residuos no apilados - Fuego en retroceso

T3: Residuos no apilados - Fuego frontal

T4: Residuos no apilados - Fuego por flancos

T5: Residuos no apilados - Sin quema "control"

T6: Extracción de residuos (diámetro > a $3 \mathrm{~cm}$ ) - Sin quema

(Fuente: FRANCKE, 1989)

Cuadro 2

RELACIÓN CT/NT EN PREDIO LA COLCHA (SUELO ARCILLOSO)

\begin{tabular}{|c|c|c|c|c|c|c|}
\hline \multirow{2}{*}{$\begin{array}{c}\text { Estrata } \\
(\mathrm{cm})\end{array}$} & \multicolumn{6}{|c|}{ Tratamiento } \\
\cline { 2 - 6 } & $\mathrm{T} 1$ & $\mathrm{~T} 2$ & $\mathrm{~T} 3$ & $\mathrm{~T} 4$ & $\mathrm{~T} 5$ & T6 \\
\hline Orgánica & 15,0 & 13,9 & 13,1 & 11,0 & 20,5 & 13,1 \\
\hline $0-5$ & 17,5 & 21,8 & 18,6 & 15,3 & 27,4 & 30,6 \\
\hline $5-10$ & 23,0 & 17,2 & 15,9 & 17,8 & 18,1 & 16,3 \\
\hline $10-20$ & 17,1 & 16,3 & 20,0 & 18,3 & 38,8 & 28,9 \\
\hline
\end{tabular}

T1: Residuos apilados - Fuego en retroceso

$\mathrm{T} 2$ : Residuos no apilados - Fuego en retroceso

T3: Residuos no apilados - Fuego frontal

T4: Residuos no apilados - Fuego por flancos

T5: Residuos no apilados - Sin quema "control"

T6: Extracción de residuos (diámetro > a $3 \mathrm{~cm}$ ) - Sin quema

(Fuente: FRANCKE, 1989) 
En los dos tipos de suelos, por efecto del fuego, en los primeros $20 \mathrm{~cm}$ de profundidad del suelo la relación CT/NT es casi siempre inferior a 20. De acuerdo a lo señalado por OPAZO (1994), en este caso se produciría una mineralización más rápida del nitrógeno.

En los tratamientos que no consideran la quema, en el suelo arcilloso en general la relación CT/NT es mayor a 20, salvo entre los 5 y $10 \mathrm{~cm}$, en cambio en el arenoso está cercana a valores de equilibrio, de acuerdo a lo señalado por OPAZO (1994).

La cantidad y naturaleza de la materia orgánica va a influir en los procesos de desnitrificación. Se ha comprobado que el carbónico orgánico soluble en agua y el carbono mineralizable tienen una alta relación con la capacidad de desnitrificación (OPAZO, 1994).

\section{REFERENCIAS BIBLIOGRÁFICAS}

Adams, M.; Iser, J.; Keleher, A.; and Cheal, D. 1994. Nitrogen and Phosphorus Avalaibility and the Role of Fire in Heathlands at Wilsons Promontory. Australian Journal of Botany 42 (3): 269 281 .

Altieri, M. y Rodriguez, J., 1975. Acción Ecológica del Fuego en el Matorral Mediterráneo de Chile, en Rinconada de Maipú. Tesis Facultad de Agronomía, Universidad de Chile. Santiago, Chile.

Barbour, M.; Burk, J.; and Pitts, W. 1987. Terrestrial Plant Ecology. The Benjamin/Cummings Publishing Company, Inc. California, USA. 634 p.

Donoso, C., 1992. Ecología Forestal, el Bosque y su Medioambiente. Tercera edición. Editorial Universitaria. Santiago, Chile. 369 p.

Feller, M. and Kimmins, J. 1984. Effects of Clearcutting and Slash Burning on Streamwater Chemistry and Watershed Nutrient Budgets in Southwestern British Columbia. Water Resources Research 20 (1): 29-40.

Francke, S., 1989. Efectos de los Diferentes Métodos de Utilización y Manejo de Residuos de Explotación en los Rendimientos de la Segunda Rotación de Pino Insigne. Informe Integrado Anual de Suelos, Parte II Efecto en Determinadas Propiedades Químicas del Suelo y Sobre la Reserva de Nutrientes del Suelo Mineral en Relación al Estado Nutricional y Rendimiento de Plantaciones de Pino Insigne en Segunda Rotación. Instituto Forestal. Santiago, Chile. 122 p.

Francke, S., 1991. Efectos del Manejo de Residuos de Explotación en el Suelo y Crecimiento Inicial de Plantaciones de Pinus radiata, en las Series de Suelo Coreo (Zona Arenales) y Colico (Zona Arauco). Documento Técnico 52, Chile Forestal. CONAF. Santiago, Chile. 8 p.

Francke, S., 1992. Efectos del Manejo de Residuos de Explotación en el Suelo y Crecimiento Inicial de Plantaciones de Pinus radiata. Documento Técnico 67, Chile Forestal. CONAF. Santiago, Chile. 8 p.

Hawley, R. y Smith, D. 1972. Silvicultura Práctica. Ediciones Omega. Barcelona, España. 544 p. 
Kozlowski, T.; Kramer, P.; and Pallardy, S., 1991. The Phisiological Ecology of Woody Plants. Academic Press, Inc. San Diego, USA. 657 p.

Kutiel, P. and Naveh, Z. 1987. The Effect of Fire on Nutrients in a Pine Forest Soil. Plant and Soil 104 (2): 267-274.

Kutiel, P. and Shaviv, A. 1989. Effect of Simulated Forest Fire on the Availability of N and P in Mediterranean Soils. Plant and Soil 120 (1): 57-63.

Landsberg, J. 1986. Physiological Ecology of Forest Production. Academic Press, Sidney, Australia. 198 p.

Leitch, C.J.; Flinn, D.W.; and Graaff, R.H.M. Van De 1983. Erosion and Nutrient Loss Resulting From Ash Wednesday (February 1983) Wildfires: A Case Study. Australian Forestry 46 (3): 173-180.

Marion, G.; Moreno, J.; and Oechel, W. 1991. Fire Severity, Ash Deposition, and Clipping Effects on Soil Nutrients in Chaparral. Soil Sci. Soc. Am. J. 55 (1): 235-249.

Opazo, J., 1994. Fertilidad de suelos. In: Suelos, una Visión Actualizada del Recurso. Editor Wilfredo Vera. Publicaciones Misceláneas Agrícolas $N^{\circ} 38$. Facultad de Ciencias Agrarias y Forestales, Universidad de Chile. Santiago, Chile. 155-195.

Peña, L., 1994. Erosión y Conservación de Suelos. In: Suelos, una Visión Actualizada del Recurso. Editor Wilfredo Vera. Publicaciones Misceláneas Agrícolas $\mathrm{N}^{\circ} 38$. Facultad de Ciencias Agrarias y Forestales, Universidad de Chile. Santiago, Chile. 215-240.

Pritchett, W. and Fisher, R. 1987. Properties and Management of Forest Soil. John Wiley and Sons. New York, USA. 494 p.

Spurr, S. and Barnes, B., 1980. Forest Ecology. Third Edition. John Wiley and Sons. New York, U.S.A. 687 p.

Toro, J., 1987. Efecto de los Diferentes Métodos de Utilización y Manejo de Residuos de Explotación en los Rendimientos de la Segunda Rotación de Pino Insigne. Informe Anual, Parte II Efecto Sobre la Reserva de Nutrientes del Suelo. Fac. Ciencias Agrarias y Forestales, Universidad de Chile; Instituto Forestal, CORFO. Santiago, Chile. 49 p.

Wells, C., 1971. Effects of Prescribed Burning on Soil Chemical Properties and Nutrient Availability. In: Prescribed Burning Symposium Proceedings. U.S.D.A. Forest Service Southeastern Forest Experiment Station. Asheville, U.S.A. 86-99.

Wells, C.; Campell R. E.; Debano, L. F.; Lewis, C. E.; Frederickson, R. L.; Franklin, E. C.; Froelich, R. C.; and Dunn, P. H. 1979. Effects of Fire on Soil, A State of Knowledge Review. U.S.D.A. Forest Service. Washington, U.S.A. 33 p. 